\title{
Effects of Soothing Liver and Invigorating Spleen Recipes on the IKK $\beta$-NF- $\kappa$ B Signaling Pathway in Kupffer Cells of Nonalcoholic Steatohepatitis Rats
}

\author{
Xiang-Wen Gong, Yong-Jian Xu, Qin-He Yang, Yin-Ji Liang, \\ Yu-Pei Zhang, Guan-Long Wang, and Yuan-Yuan Li \\ Department of Traditional Chinese Medicine, Medical College of Jinan University, 601 Huangpu Road West, Guangzhou, \\ Guangdong 510632, China
}

Correspondence should be addressed to Qin-He Yang; tyangqh@jnu.edu.cn

Received 16 February 2015; Revised 19 May 2015; Accepted 31 May 2015

Academic Editor: Bashar Saad

Copyright (C) 2015 Xiang-Wen Gong et al. This is an open access article distributed under the Creative Commons Attribution License, which permits unrestricted use, distribution, and reproduction in any medium, provided the original work is properly cited.

\begin{abstract}
This study investigates the effect of soothing liver and invigorating spleen recipes on steatohepatitis examining the IKK $\beta$-NF- $\kappa \mathrm{B}$ signaling pathway in KCs of NASH rats. SD male rats were randomly divided into 8 groups, and the NASH model was induced by a high-fat diet (HFD). After 26 weeks, liver tissue was examined in H\&E stained sections and liver function was monitored biochemically. KCs were isolated by Seglen's method, with some modifications. The mRNA and protein expression of the IKK $\beta$ NF- $\kappa$ B signaling pathway components was examined by quantitative PCR and Western blotting. The results show that the high-fat diet induced NASH in the rats, and the soothing liver recipe and invigorating spleen recipe decreased the levels of TNF- $\alpha$, IL1 , and IL- 6 in KCs, as well as inhibiting the mRNA and protein expression of the IKK $\beta$-NF- $\kappa \mathrm{B}$ signaling pathway components. In conclusion, the experiment indicated the importance of the IKK $\beta$-NF- $\kappa \mathrm{B}$ signaling pathway in KCs for the anti-inflammatory effects of the soothing liver and invigorating spleen recipes.
\end{abstract}

\section{Introduction}

Nonalcoholic fatty liver disease (NAFLD) encompasses a spectrum of diseases ranging from simple hepatic steatosis to the aggressive condition, nonalcoholic steatohepatitis, whereas NASH is an aggressive liver disease which leads to advanced fibrosis, cirrhosis, and even hepatic failure [1]. NAFLD is also a strong independent predictor of cardiovascular disease and may play a central role in the cardiovascular risks of the metabolic syndrome [2]. Epidemiological studies suggest that NAFLD has become the most common cause of chronic liver disease worldwide among children and adolescents, with a prevalence in the west of approximately $15 \sim 35 \%$ [3]. The overall prevalence of NAFLD in China was $20.09 \%$ (17.95 22.31\%) [4]. Hence, studies of the prevention and treatment of NAFLD are important.

Because the pathogenesis of NAFLD is incompletely characterized, there is no consensus regarding the most effective and appropriate pharmacologic therapy [5]. Herbal treatment for NAFLD has received increasing attention in recent decades due to its wide availability, minimal side effects, proven therapeutic mechanisms, and benefits [6]. Evidencebased medicine supports this point of view [7]. Based on TCM theory, liver stagnation and spleen deficiency were principal pathogenesis of NAFLD. Following the principles of "prescription-syndrome correspondence," the important treatment would be soothing liver and invigorating spleen recipes [8]. Clinical and experiment studies showed that soothing liver and invigorating spleen recipes could effectively treat NAFLD $[9,10]$.

The soothing liver recipe (Chaihu-Shugan-San) is a classical formula from "Jingyue Quanshu" that was written by Jingyue Zhang, a famous Ming dynasty physician, in China in 1640 A.D. The invigorating spleen recipe (Shen-ling-bai-zhuSan) is also a famous classical formula recorded in "Taiping Huimin Heji Ju Fang" that was written by the Imperial 
Medical Bureau of the Song Dynasty in 1078 A.D. The invigorating spleen recipe is recognized in the widely used official Chinese Pharmacopoeia. According to TCM theory, the soothing liver recipe dredges liver qi and enhances blood circulation and is prescribed mainly for liver qi stasis. The invigorating spleen recipe invigorates the spleen and stomach qi, which it is mainly used to restore. Chaihu-Shugan-San and Shen-ling-bai-zhu-San have a significant effect on dredging by the liver and spleen deficiency.

IKK $\beta$-NF- $\kappa \mathrm{B}$ is an important redox-sensitive and proinflammatory transcription factor that plays a critical role in the regulation of a variety of genes important in cellular responses [11]. Our previous study showed that rats fed a high-fat diet for 16 weeks developed NAFLD and that I kappa $\mathrm{B}$ kinase $\beta(\operatorname{IKK} \beta)$, phospho-IKK $\beta(\mathrm{p}-\mathrm{IKK} \beta)$, and nuclear factor $-\kappa \mathrm{B}(\mathrm{NF}-\kappa \mathrm{B})$ were highly expressed in their KCs, implying a relationship between NAFLD and the IKK $\beta$ $\mathrm{NF}-\kappa \mathrm{B}$ pathway [12]. We next asked whether rats fed a HFD for 26 weeks would develop NASH and, in this paper, we study the effects of soothing liver and invigorating spleen recipes on inflammatory markers and proteins involved in IKK $\beta-N F-\kappa B$ p 65 pathway in KCs to explore some of the underlying mechanisms involved. To explore the evolution of the pathology, we also examined the inflammatory changes that accompany NAFLD at different periods.

\section{Materials and Methods}

2.1. Preparation of Soothing Liver and Invigorating Spleen Recipes. The soothing liver recipe is composed of seven Chinese herbs: Bupleuri radix, Citri reticulatae pericarpium, Chuanxiong Rhizoma, Cyperi Rhizoma, Bitter Orange Aurantii Fructus, Paeonia lactiflora Pall, and Glycyrrhizae radix, in a traditional dose ratio of $6: 6: 5: 5: 5: 5: 3$. The invigorating spleen recipe includes Dolichos lablab, Atractylodes macrocephala Koidz, Poria cocos (Schw.) Wolf, Glycyrrhizae radix, Platycodi radix, Sulphur, Panax ginseng C. A. Mey, Amomum villosum Lour, Dioscorea opposita Thunb, and Semen Coicisin a ratio of $5: 5: 5: 5: 4: 3: 3: 3: 2: 2$. The integrated recipe is a mixture of CSGS and SLBZ in a $1: 1$ ratio. All Chinese medicines were purchased as formula granules from Shenzhen Sanjiu Medical Co., Ltd. (1005001S). The formula granules were dissolved in distilled water and stored at $-4^{\circ} \mathrm{C}$ in a refrigerator.

2.2. Animals. $120 \mathrm{SD}$ rats (6 weeks old, $200 \mathrm{~g} \pm 20 \mathrm{~g}$ ) were obtained from the Laboratory Animal Research Center of Guangzhou University of Traditional Chinese Medicine (China, Animal License Key no. 0107792; License no. SCXK (Yue) 2008-0020). The use of animals in this study was approved by the Ethics Committee of Medical College of Jinan University. The rats were separately housed in the Animal Administration Laboratory, Jinan University, at a controlled temperature $\left(24^{\circ} \mathrm{C} \pm 2^{\circ} \mathrm{C}\right)$ and humidity $(70 \% \pm 10 \%)$ with a $12 \mathrm{~h}$ light and $12 \mathrm{~h}$ dark cycle, with free access to food and water.
2.3. Grouping and Modeling. After $7 \mathrm{~d}$ for adaptation, the rats were randomly divided into 8 groups, each of 15 rats (liver samples were taken from 9 rats of each group and KCs were isolated from the remaining 6). The groups comprised normal, model, low-dose soothing liver recipe (L-SLG), highdose soothing liver recipe (H-SLG), low-dose invigorating spleen recipe (L-ISG), high-dose invigorating spleen recipe (H-ISG), low-dose integrated recipe (L-IG), and high-dose integrated recipe (H-IG) group. NASH was reproduced in our rats by our previously reported method [13] with some minor modifications. The normal group of rats had free access to a normal chow diet, and the model group of rats were fed a HFD composed of regular chow $88 \%$, axungia porci $10 \%$, cholesterol $1.5 \%$, and bile salt $0.5 \%$. All recipes were given by gastrogavage [14] at $8 \mathrm{am}$ at $1 \mathrm{~mL} / 100 \mathrm{~g}$ body weight for the low-dose groups (equivalent to the human dose) and 3 times this volume for the high-dose groups. Rats in the normal and model groups were fed with the same dose of distilled water once daily at 8:00 am. All treatments lasted for 26 weeks.

2.4. Histopathological Examination of Liver Tissue. Rats were anesthetized by intraperitoneal injection of $3 \%$ pentobarbital $(0.2 \mathrm{~mL} / 100 \mathrm{~g}$ body weight) and a portion (approximately $1 \mathrm{~cm} \times 1 \mathrm{~cm} \times 0.5 \mathrm{~cm}$ ) of liver approximately $0.5 \mathrm{~cm}$ from the edge of the right hepatic lobule was removed and paraffinembedded for sectioning and hematoxylin-eosin ( $\mathrm{H} \& \mathrm{E})$ staining. The steatosis, fibrosis, and inflammation of NASH were identified by light microscopy.

2.5. Biochemical Test in Serum. Liver tissues were placed in isopropanol and homogenized with a TissueLyser-II homogenizer, centrifuged at $3000 \times \mathrm{g}, 4^{\circ} \mathrm{C}$ for $10 \mathrm{~min}$, when the clear supernatants were collected. Levels of alanine aminotransferase (ALT), aspartate aminotransferase (AST), and the AST/ALT ratio in the serum were determined with automatic biochemical analyzer.

2.6. Separation and Identification of KCs. KCs were isolated and identified from 6 rats in each group as previously described [15], and some modifications were made. After rats were anesthetized, the liver was perfused in situ with $200 \mathrm{~mL}$ $0.5 \mathrm{mmol} / \mathrm{L}$ Ethylene Glycol Tetraacetic Acid (EGTA) in DHanks at $20 \mathrm{~mL} / \mathrm{min}, 37^{\circ} \mathrm{C}$, until the color of the liver changed into amber. Then the liver was transferred to a culture dish and was perfused ex situ with $0.03 \%$ collagenase IV in Hanks, which contained $5 \mathrm{mmol} / \mathrm{L}$ calcium ions and was preheated to $37^{\circ} \mathrm{C}$, at $20 \mathrm{~mL} / \mathrm{min}$ in a recirculating fashion for $15 \mathrm{~min}$. The liver was then placed in $10 \mathrm{~mL}$ RPMI-1640 culture medium containing $10 \%$ fetal calf serum (FBS). The capsule and fibrous tissue were removed, and the remaining tissue was cut into small pieces. After the obtained liver homogenate was filtered through a $200 \mu \mathrm{m}$ and $300 \mu \mathrm{m}$ nylon mesh, the cell suspension was centrifuged at $350 \mathrm{rpm}, 4^{\circ} \mathrm{C}$, for $3 \mathrm{~min}$ and clear supernatant was collected in another tube and centrifuged at $1050 \mathrm{rpm}, 4^{\circ} \mathrm{C}$, for $10 \mathrm{~min}$. The cell pellet was subsequently resuspended in RPMI-1640 containing $10 \%$ FBS. 
TABle 1: Primer sequences, annealing temperatures, and length of products in real-time PCR.

\begin{tabular}{lll}
\hline Gene & Forward & Backward \\
\hline GAPDH & GATCCCGCTAACATCAAATG & GAGGGAGTTGTCATATTTCTC \\
IKK $\beta$ & GAGAAGAAAGTGCGGGTGATTTACT & GAGCCTCACCACCTCTTCTACTTT \\
NF- $\kappa$ B & GTGGGCAAGCACTGTGAGGA & TCATCCGTGCTTCCAGTGTTTC \\
\hline
\end{tabular}

Then some $15 \mathrm{~mL}$ centrifuge tubes were carefully laid into $2.5 \mathrm{~mL} 24 \%$ Nycodenz working solution at the bottom, $2.5 \mathrm{~mL} 11 \%$ Nycodenz working solution in the middle layer, and $2.5 \mathrm{~mL}$ the cell suspension at the top. Then it was centrifuged at $2500 \mathrm{rpm}, 4^{\circ} \mathrm{C}$, for $15 \mathrm{~min}$. KCs cloud appearance between the $11 \%$ Nycodenz layer and $24 \%$ Nycodenz layer was collected into another $15 \mathrm{~mL}$ tube, resuspended in GBSS, and then centrifuged twice at $1050 \mathrm{rpm}, 4^{\circ} \mathrm{C}$, for $15 \mathrm{~min}$. The cell pellet was then resuspended and seeded on a culture dish at a density of $2-5 \times 10^{6}$ cells $/ \mathrm{mL}$ with RPMI-1640 containing $10 \% \mathrm{FBS}$ and incubated in a $5 \% \mathrm{CO}_{2}$ atmosphere for $30 \mathrm{~min}$ at $37^{\circ} \mathrm{C}$. By further using adhesion purification, KC purity was improved, and cell viability was tested by trypan blue dye exclusion.

2.7. Determination of Inflammatory Cytokines in KCs. KCs from each group were isolated and identified. The cells were then centrifuged, washed 3 times in PBS, adjusted to $1 \times 10^{6} / \mathrm{mL}$, and sonicated at $4^{\circ} \mathrm{C}$ for $15 \mathrm{~min}$ at $10000 \mathrm{rpm}$. Clear supernatants were used for the cytokine measurements. TNF- $\alpha$, IL-1, and IL- 6 were measured by enzymelinked immunosorbent assay (ELISA) using ShangHai ExCell Biotechnology Co., Ltd. (China) kits: 24122012-002 for TNF$\alpha$, ZGAHBZAB01 for IL-1, and ZIBIBZAB02 for IL-6.

2.8. Determination of IKK $\beta$ and NF- $\kappa B$ p 65 mRNA Expression in KCs. Total RNA was extracted from KCs using TRIZOL Reagent. The integrity of each RNA sample was evaluated by agarose gel electrophoresis, its purity and concentration were assayed, and then the total RNA was reverse transcribed to $\mathrm{CDNA}$. Using the gene sequences for IKK $\beta$, NF- $\kappa \mathrm{B}$ p 65 , and glyceraldehyde-3-phosphate dehydrogenase (GAPDH) in Genebank, the primers were designed and synthesized by Shanghai Generay Biological Engineering Co., Ltd. GAPDH was used as the reference gene. The reaction conditions were as follows: (1) predenaturation for $10 \mathrm{~s}$ at $95^{\circ} \mathrm{C}$, (2) denaturation for $5 \mathrm{~s}$ at $95^{\circ} \mathrm{C}$, (3) $\mathrm{GAPDH} 55^{\circ} \mathrm{C}, \operatorname{IKK} \beta 60^{\circ} \mathrm{C}$, NF- $\kappa \mathrm{B} 62^{\circ} \mathrm{C}$, renaturation for $20 \mathrm{~s}$, (4) extension for $40 \mathrm{~s}$ at $60^{\circ} \mathrm{C}$, (2)-(4) being repeated 39 times, and (5) analysis of the solubility curve, $72^{\circ} \mathrm{C}$ to $95^{\circ} \mathrm{C}$ for 5-10 s. After the reaction was finished, the results were analyzed using Opticon Monitor 3.1 software, and the $2^{-\Delta \Delta \mathrm{Ct}}$ formula was used for relative quantification (Table 1).

2.9. Analysis of IKK $\beta$, $p-I K K \beta$, and NF- $\kappa B$ p 65 Protein Expression in KCs. The IKK $\beta$, p-IKK $\beta, \mathrm{NF}-\kappa \mathrm{B}$ p65, and GAPDH proteins in KCs were measured by Western blotting. GAPDH was used as an internal control. KCs were lysed in RIPA lysis buffer and centrifuged at $12000 \mathrm{rpm}$ for $5 \mathrm{~min}$ at $4^{\circ} \mathrm{C}$, when the supernatants were collected. The concentration of supernatant protein was determined by a BCA protein assay. Protein preparations were subjected to $10 \%$ SDSPAGE and transferred to a polyvinylidene difluoride (PVDF) membrane. After transfer, the membrane was blocked in 5\% skim milk in Tris-Buffered Saline Tween-20 (TBST) and incubated overnight at $4^{\circ} \mathrm{C}$ with specific primary antibodies. The IKK $\beta$ antibody (no. 0002), anti-phosphor-IKK $\beta$ antibody (no. 0013), NF- $\kappa \mathrm{B}$ p65 antibody (no. 0006), and GAPDH antibody were purchased from Cell Signaling Technology (USA). Then, horseradish peroxidase (HRP) conjugated goatanti-rabbit antibody was added and incubated at room temperature for $1 \mathrm{~h}$. After being washed for three times in TBST, the PVDF membrane was put into developer and exposed to X-ray film. The films were scanned and analyzed by a gel image processing system.

2.10. Statistical Analysis. Statistical analyses were performed by using SPSS 13.0. The values were presented as the mean \pm standard. One-way analysis of variance (one-way ANOVA) and Tukey's test were used to determine the statistical significance of the differences. $P$ values less than 0.05 were considered significant.

\section{Results}

3.1. Histopathological Changes. Sections were stained with H\&E staining. As shown in Figure 1, hepatocyte nuclei were blue, and cytoplasm was uniformly red-stained, with less or no adipose hollow space. The hepatic lobule and liver rope both had clear structures and regular arrangement. There was no point necrosis or soakage of inflammatory cell soakage. In the model group, the central vein and portal area appeared as a diffuse adipose hollow space. Hepatocytes had obvious tumefaction or enlargement or even ballooning. Substantial adipose hollow space was observed in the cytoplasm. Some small hollow spaces were converted into a larger space. The narrow hepatic sinusoidal and disorder liver rope were observed. It was difficult to find the normal hepatocytes. Inflammatory infiltrates were found in the hepatic lobule and portal areas are scant. Compared with the model group, the structure arrangement, morphological features, macrovesicular lipid droplets, ballooning, inflammatory infiltrates, and spots necrosis improved by various degrees in all drug therapy groups. H-IG had the most significant impact on liver tissue histopathology.

3.2. Changes of ALT, AST, and AST/ALT in Serum. The ALT results showed no differences between the normal and model groups, and there were no obvious differences in the serum ALT levels of the treatment groups $(P>0.05)$. The 


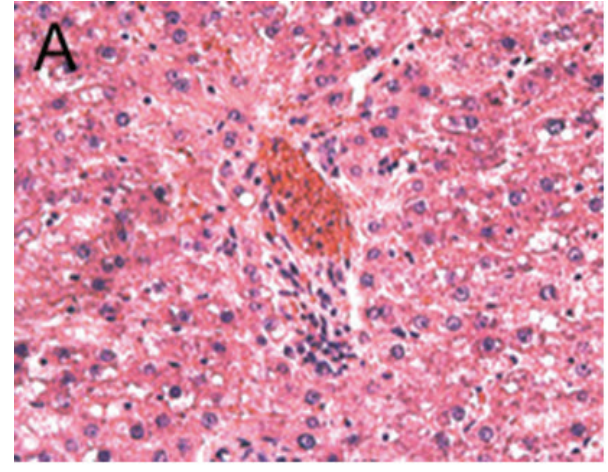

(a)

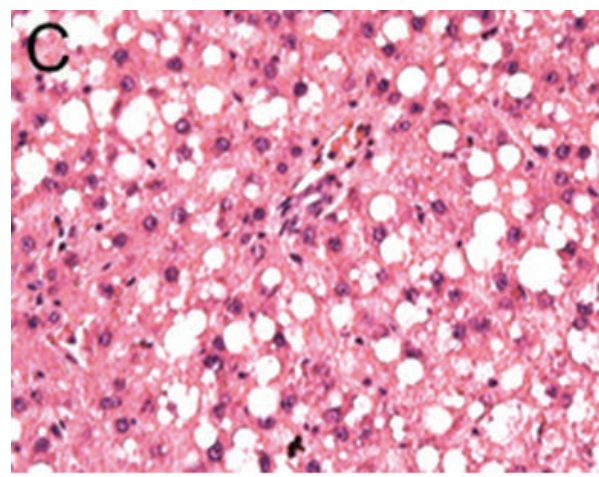

(c)

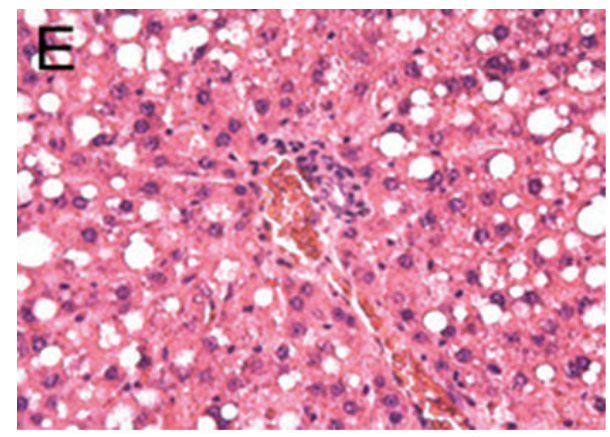

(e)

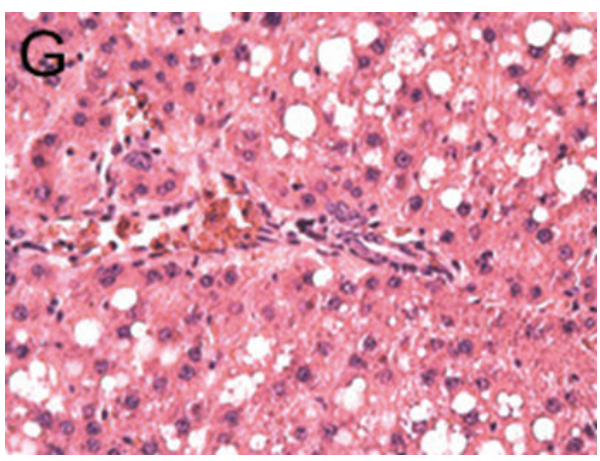

(g)

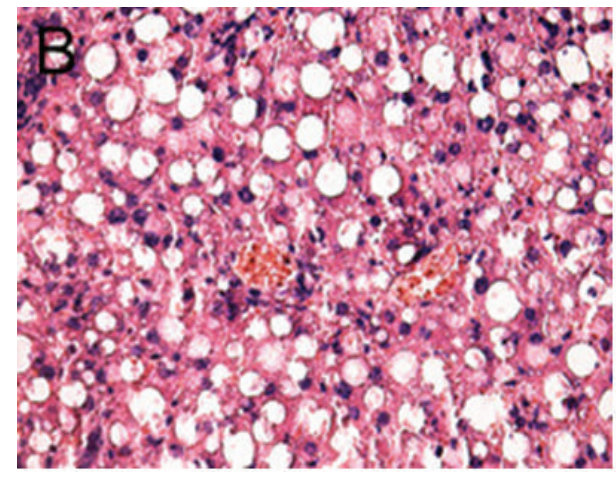

(b)

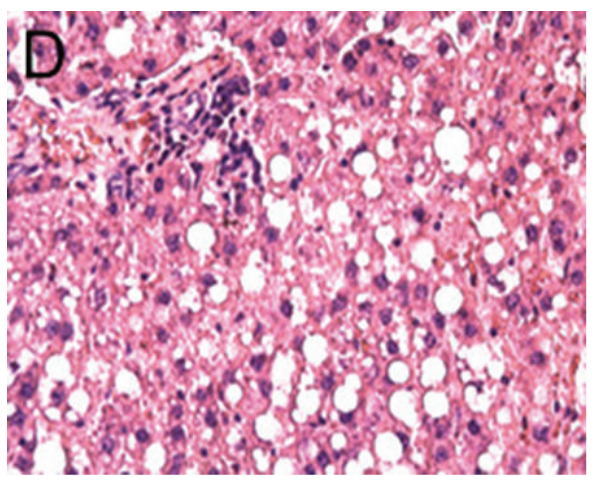

(d)

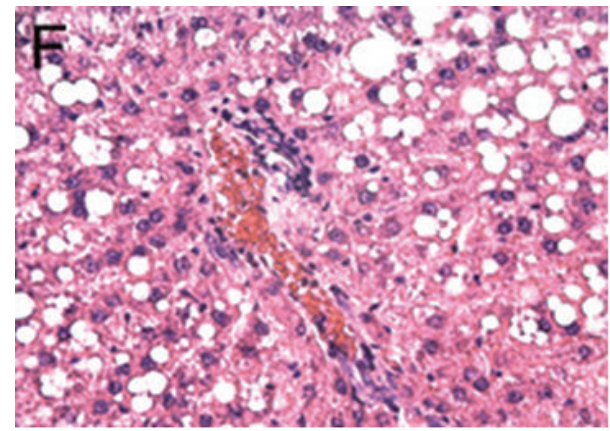

(f)

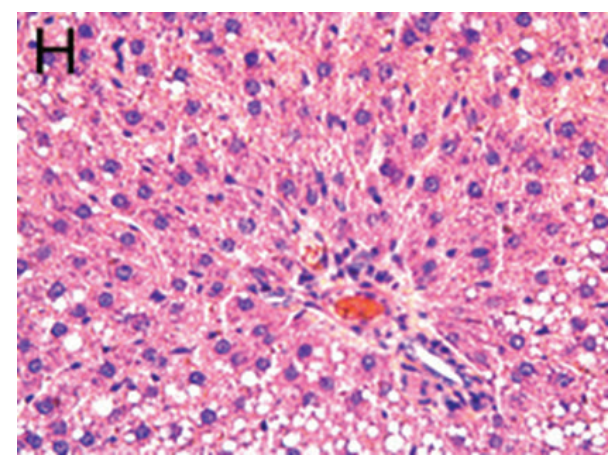

(h)

FIGURE 1: Histological changes of liver sections in different groups (HE stain $\times 200$ ). (a) Normal group, (b) model group, (c) L-SLG, (d) H-SLG, (e) L-ISG, (f) H-ISG, (g) L-IG, and (h) H-IG. 


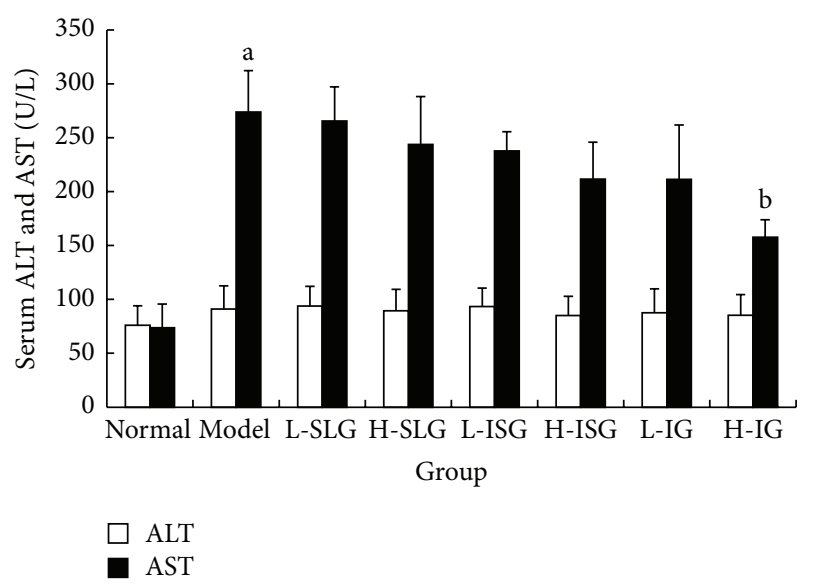

FIgURE 2: Levels of ALT and AST in serum were determined. Rats were fed with normal chow diet or HFD with or without CSS and SLBZS for 26 weeks. The values were expressed as mean \pm S.E.M. 9 rats per group. ${ }^{\mathrm{a}} \mathrm{P}<0.01$ versus normal group; ${ }^{\mathrm{b}} \mathrm{P}<0.01$ versus model group.

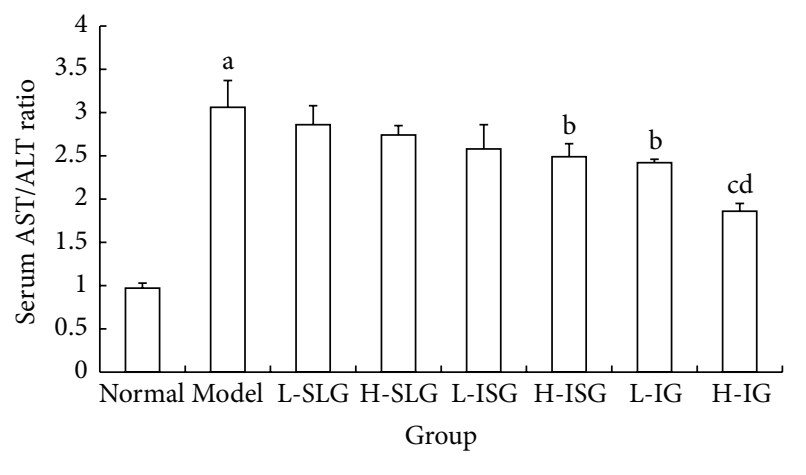

AST/ALT

FIGURE 3: AST/ALT ratio in serum was computed. Rats were fed with normal chow diet or HFD with or without CSS and SLBZS for 26 weeks. The values were expressed as mean \pm S.E.M. 9 rats per group. ${ }^{\mathrm{a}} P<0.01$ versus normal group; ${ }^{\mathrm{b}} P<0.05,{ }^{\mathrm{c}} P<0.01$ versus model AST/ALT; ${ }^{\mathrm{d}} P<0.01$ versus L-SLG, H-SLG, L-ISG, H-IS, and L-IG.

serum AST level and AST/ALT ratio of the model group were obviously increased $(P<0.01)$ compared with normal group. Compared with model group, the H-IG group had a significantly decreased level of AST $(P<0.05)$. The AST/ALT ratios of the H-SG, H-IG, and L-IG were all decreased $(P<$ $0.01, P<0.05)$, as shown in Figures 2 and 3.

3.3. The Population, Purity, and Viability of KCs. KCs were isolated from 6 rats in each group by collagenase perfusion as described. After $3 \mathrm{~h}$ incubation at $37^{\circ} \mathrm{C}$, the cells were washed 3 times with PBS and nonadherent cells were washed off. The KCs viability was $>95 \%$ (as tested by trypan blue dye exclusion). The purity of KCs was $91.21 \%$ (as assessed by flow cytometry method using a Lysozyme antibody), as shown in Figures 4 and 5.

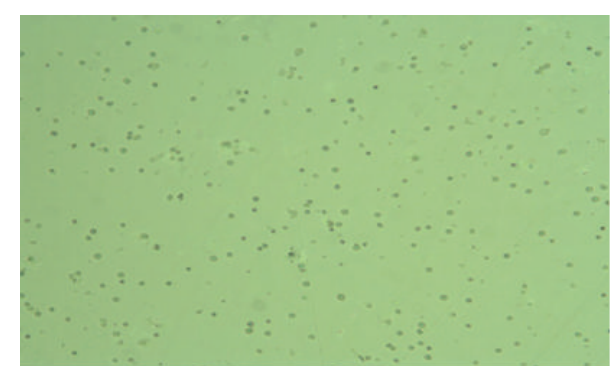

FIGURE 4: Isolated rat KCs cultured for $3 \mathrm{~h}(\times 100)$.

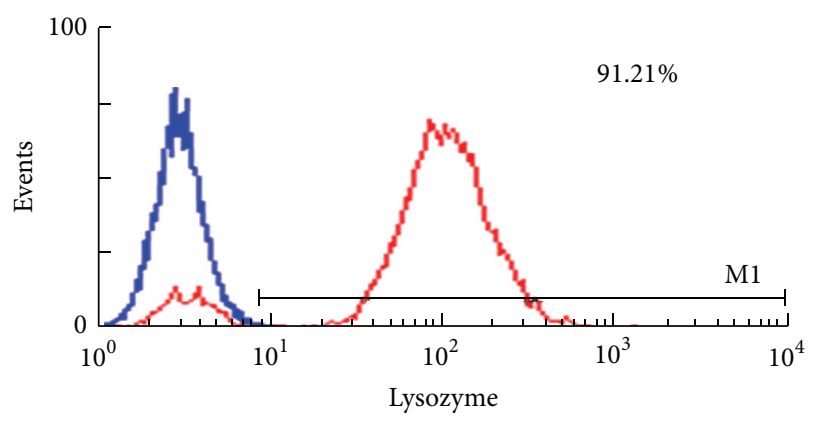

FIgURE 5: The purity of KCs identified by flow cytometer. Isolated rat KCs positive for lysozyme were more than $91.21 \%$.

3.4. Changes of Inflammatory Cytokines in KCs. Higher levels of TNF- $\alpha$, IL-1, and IL- 6 were observed in KCs of the model group compared with those of the normal group $(P<0.01)$. Compared with the model group, the H-ISG, H-IG, and LIG groups showed significant decreases in TNF- $\alpha$ and IL-6 $(P<0.01$ or $P<0.05)$, and the H-ISG and H-IG had clearly lower levels of IL-1 $(P<0.01$ or $P<0.05)$. The results showed that the high dose of both the invigorating spleen recipe and integrated recipes reduced the TNF- $\alpha$, IL-1, and IL- 6 levels of liver inflammatory cytokine in rats with NASH induced by HFD, as shown in Figure 6.

3.5. The IKK $\beta$ and NF- $\kappa B$ Expression in KCs. Compared with the normal rats, the levels of IKK $\beta$ and NF- $\kappa$ B mRNA in KCs of the model group increased 20.56-fold and 16.29-fold, $(P<$ $0.01)$. The H-CG, H-SG, H-IG, and L-IG treated animals had lower expression levels of IKK $\beta$ and NF- $\kappa$ B mRNA than the model rats $(P<0.01, P<0.05)$. The expression levels of IKK $\beta$ and NF- $\kappa \mathrm{B}$ mRNA in the H-IG were obviously lower than those of the H-SG and L-IG $(P<0.05)$, as shown in Table 2.

3.6. Expression of $I K K \beta, p-I K K \beta$, and NF- $\kappa B$ p65 Proteins in KCs. To explore the mechanism of the anti-inflammatory effect of soothing liver and invigorating spleen recipes in the KCs of NASH rats, we assayed three important proteins, $\operatorname{IKK} \beta$, p-IKK $\beta$, and NF- $\kappa \mathrm{B}$, which participate importantly in the NF- $\kappa \mathrm{B}$ p65 signaling pathway to an inflammatory response, as shown in Figure 7(a). The expression levels of $\mathrm{IKK} \beta$, p- IKK $\beta$, and NF- $\kappa \mathrm{B}$ p65 in the model group were significantly higher than those in the normal control group $(P<0.01$, Figure $7(b))$. Compared to the model group, the 
TABLE 2: Expression of IKK $\beta$ and NF- $\kappa$ B mRNA in KCs $(\bar{x} \pm s, n=$ $6)$.

\begin{tabular}{lcc}
\hline & $\begin{array}{c}2^{-\Delta \Delta \mathrm{CT}} \text { IKK } \beta \text { Rel. to } \\
\text { control }\end{array}$ & $\begin{array}{c}2^{-\Delta \Delta \mathrm{CT}} \text { NF- } \kappa \mathrm{B} \text { p65Rel. } \\
\text { to control }\end{array}$ \\
\hline Normal & $1(0.25-4.09)$ & $1(0.39-2.65)$ \\
Model & $20.56(4.56-34.05)^{\mathrm{a}}$ & $16.29(2.77-48.16)^{\mathrm{a}}$ \\
L-SLG & $17.99(1.89-30.69)$ & $14.43(2.33-31.12)$ \\
H-SLG & $14.34(3.51-23.43)^{\mathrm{b}}$ & $10.39(2.15-22.31)^{\mathrm{b}}$ \\
L-ISG & $15.63(7.31-28.05)$ & $12.07(7.06-15.78)$ \\
H-ISG & $10.32(3.83-23.91)^{\mathrm{c}}$ & $9.15(4.43-27.10)^{\mathrm{c}}$ \\
L-IG & $12.09(4.75-20.11)^{\mathrm{b}}$ & $9.85(2.56-18.90)^{\mathrm{b}}$ \\
H-IG & $6.55(1.80-14.83)^{\mathrm{cd}}$ & $4.76(0.55-12.47)^{\mathrm{cd}}$ \\
\hline
\end{tabular}

Expression of IKK $\beta$ and NF- $\kappa$ B mRNA in KCs was determined by Q-PCR. Rats were fed with normal chow diet or HFD with or without CSS and SLBZS for 26 weeks. The values were expressed as mean \pm S.E.M. of 6 rats per group. ${ }^{\mathrm{a}} P<0.01$ versus normal group; ${ }^{\mathrm{b}} P<0.05,{ }^{\mathrm{c}} P<0.01$ versus model group, ${ }^{\mathrm{d}} P<0.01$ versus $\mathrm{H}-\mathrm{ISG}$ and L-IG group.

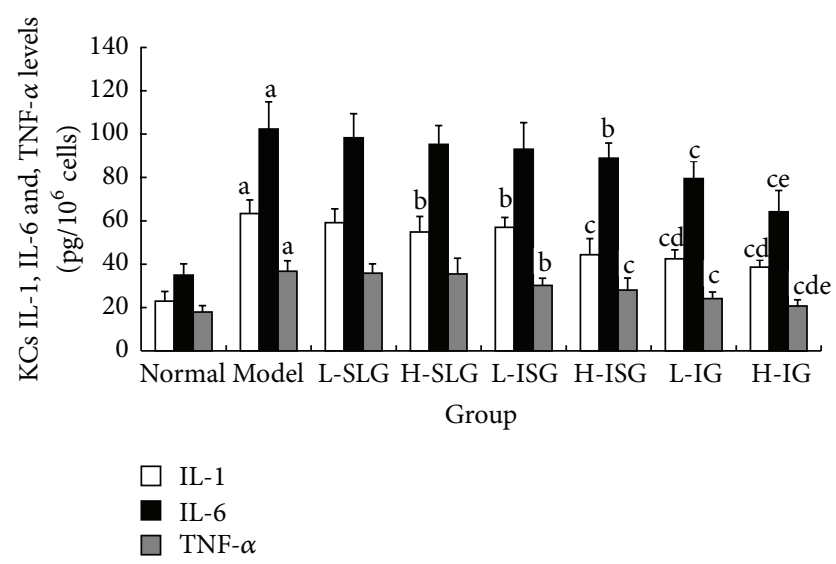

FIGURE 6: Related inflammatory cytokines of IL-1, IL-6, and TNF- $\alpha$ in KCs were determined by ELISA. Rats were fed with normal chow diet or HFD with or without CSS and SLBZS for 26 weeks. The values were expressed as mean \pm S.E.M. 6 rats per group. ${ }^{a} P<0.01$ versus normal group; ${ }^{\mathrm{b}} P<0.05,{ }^{\mathrm{c}} P<0.01$ versus model group; ${ }^{\mathrm{d}} P<0.01$ compared with H-SLG; ${ }^{\mathrm{e}} P<0.01$ compared with L-ISG.

expression levels of IKK $\beta$, p- IKK $\beta$, and NF- $\kappa \mathrm{B}$ p65 were reduced in all treatment groups $(P<0.01, P<0.05)$. We found that $\mathrm{IKK} \beta, \mathrm{p}-\mathrm{IKK} \beta$, and NF- $\kappa \mathrm{B}$ p 65 protein expression was inhibited in the H-IG more than in the H-CG, L-CG, HSG, and L-SG (Figure 7(b)).

\section{Discussion}

In our previous study, a rat model of NASH was established using 16 weeks of HFD, so as to resemble the pathogenesis of NASH in humans more closely. In this study, we showed by $\mathrm{H} \& \mathrm{E}$ staining that NASH was successfully established. In the model group, the central vein and portal areas appeared as diffuse adipose hollow spaces. The hepatocytes had obvious tumefaction, enlargement, or even ballooning. The H\&E staining also suggested that the different treatments affected the NAFLD to different degrees, with the H-IG superior to other groups.

Past studies indicated that the ALT and AST are the most useful tools for diagnosis of the chronic liver disease. Beyond these tools, the ALT/AST ratio is a prognostic parameter in patients with liver injury [16]. If hepatocytes are badly damaged and their cytoplasm and mitochondria are destroyed, AST is released into the blood and its level increases more than that of ALT, so the AST/ALT ratio increases [17]. The AST/ALT ratio has therefore become an important index for the diagnosis of NAFLD [18]. In this study, the serum AST levels and AST/ALT ratios were obviously increased $(P<0.05)$. We speculate that liver injury already exists in NAFLD rats. Combining the H\&E staining and liver function tests, we can see that the liver has sustained serious damage from inflammation. We now show that a high dose of integrated recipes protects against liver injury and moderates NASH progression $(P<0.05)$. TCM theory attributes the abnormalities in the 26 weeks' HFDinduced NASH rats to liver stagnation and spleen deficiency. Therefore, the effects of the soothing liver recipe and the invigorating spleen recipe were superior to other classical formulas.

KCs are resident hepatic macrophages that account for $80-90 \%$ of the total number of fixed tissue macrophages of the body [19]. KCs eliminate and detoxify microorganisms, endotoxins, and degenerated cells, as well as possessing other functions [20]. Therefore, KCs play an important role in liver physiological homeostasis and are intimately involved in the liver's response to infection, toxins, and various other stresses through the expression and secretion of soluble inflammatory mediators [21, 22]. KCs are associated with the proinflammatory response and produce associated cytokines such as IL-1 $\beta$, IL-12, IL-23, and TNF- $\alpha$. Cytokines act as protective mediators for the recovery of normal liver function. However, excessive activation of KCs may aggravate liver damage [23, 24]. According to the two-hit hypothesis, the second hit is an exacerbating factor such as an inflammatory cytokine. Previous studies show that inflammatory KCs play a key role in NASH [25]. In this paper, the expression of IL-6 and TNF- $\alpha$ was significantly increased in KC supernatants from the model group $(P<0.01)$ while the levels in the supernatants of the low- and high-dose integrated recipes were significantly lower than the other groups $(P<0.01, P<$ $0.05)$. These findings suggest that increases in liver TNF- $\alpha$, IL-1, and IL-6 may aggravate hepatic inflammation, necrosis, and fibrosis and that the high dose of the invigorating spleen and integrated recipes may have a favorable effect on the inflammatory reaction in the steatotic liver.

As everyone knows, excessive inflammatory cytokines such as TNF- $\alpha$, IL-1, and/or IL-6 exacerbated liver injury and promoted NASH progression in different ways [26]. The mechanisms involved remain unclear, though many reports implicate the NF- $\kappa \mathrm{B}$ signal pathway. To elucidate the regulatory mechanisms in the NF- $\kappa \mathrm{B}$ signaling pathway and the anti-inflammatory effects of the soothing liver and invigorating spleen recipes further, we examined the expression and levels of several proteins closely related to the signal 


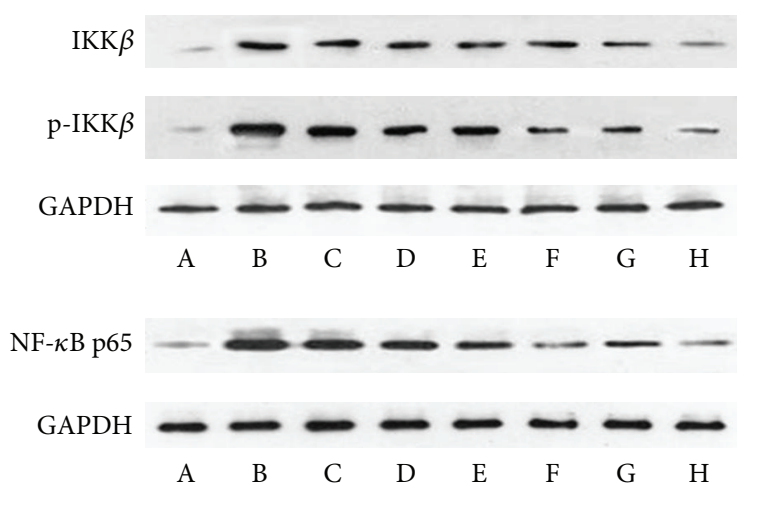

(a)

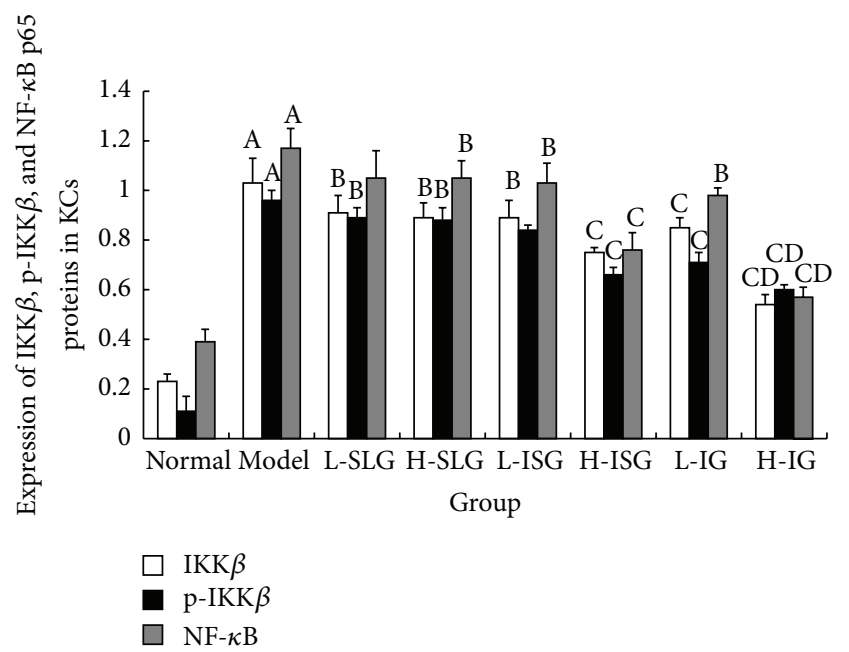

(b)

FIGURE 7: (a) Western blot of IKK $\beta$, p-IKK $\beta$, and NF- $\kappa$ B p65 proteins in KCs. A: normal group; B: model group; C: L-SLG; D: H-SLG; E: L-ISG; F: H-ISG; G: L-IG; H: H-IG. (b) Expression of IKK $\beta$, p-IKK $\beta$, and NF- $\kappa$ B p65 proteins in KCs. Rats were fed with normal chow diet or HFD with or without CSS and SLBZS for 26 weeks. The values were expressed as mean \pm S.E.M. 6 rats per group. ${ }^{A} P<0.01$ versus normal group; ${ }^{\mathrm{B}} P<0.05,{ }^{\mathrm{C}} P<0.01$ versus model group; ${ }^{\mathrm{D}} P<0.01$ versus L-SLG, H-SLG, L-ISG, H-IS, and L-IG.

transduction in the NF- $\kappa$ B pathway of KCs from NASH rats [27].

The IKK $\beta$-NF- $\kappa \mathrm{B}$ p 65 signaling pathway is an important regulator of inflammatory gene transcription involved in many chronic inflammatory diseases. In NASH, different activators of the IKKs-I $\kappa \mathrm{B}-\mathrm{NF}-\kappa \mathrm{B}$ p65 signaling pathway in $\mathrm{KCs}$ regulate the synthesis of downstream inflammatory mediators. Possible pathways are reviewed in and include [28-31] (1) LPS captured by LPS-binding protein (LBP), with the LPS-LBP complex then interacting with the membrane form of CD14 on the surface of KCs. TLR4 serves as the LPS receptor and binds MyD88 to activate IRAK-1 or IRAK-4, leading to downstream activation of the IKKs-I Kb-NF- $\kappa \mathrm{B}$ signaling pathway. (2) The TNF receptor combined with its related apoptosis structural domain protein TRADD interacts with the TNFR-2 pathway through ubiquitinated receptor interacting protein RIP and finally forms the RNFR complex with IKK $\gamma$, which leads to activation of the IKKs-I $\kappa \mathrm{B}-\mathrm{NF}-\kappa \mathrm{B}$ signaling pathway. During hepatic steatosis, inflammation, and fibrosis, hepatic NF- $\kappa \mathrm{B}$ is highly expressed, though IKK $\beta / N F-\kappa B$ pathway activation is inhibited. These findings suggest that it may be possible to delay the occurrence of inflammation and liver steatosis and insulin resistance (IR). (3) A previous study showed that, in IRF3 gene-knockout mice, activation of the IKK $\beta / \mathrm{NF}-\kappa \mathrm{B}$ signaling pathway caused severe inflammation of the liver IR and fatty degeneration.

Traditional Chinese medicine has received increasing attention as an alternative source of treatments for a variety of diseases [32]. According to TCM theory, NAFLD belongs to the Gan-Pi and Gan-Zhu. Epidemiological researches showed that the syndrome of stagnation of liver qi and spleen deficiency is one of the most common syndromes of
NAFLD in china, and the proportion is $34.7 \%$. This study suggested the clinical characteristics of Chinese NAFLD population in contemporary [33]. Therefore, the syndrome of stagnation of liver qi and spleen deficiency has become the most important syndrome in expert consensus document of NAFLD [34]. According to Chinese medicinal chemistry, the principal active components of Senlinbaizhu Powder and Chaihushugan Powder include: ferulic acid, ginsenoside, paeoniflorin, naringin, hesperidin, meranzin hydrate, neohesperidin, albiflorin, and atractylenolide, together with other drug ingredients $[35,36]$. At the same time, Chinese medicinal pharmacology has demonstrated that Chaihu-ShuganSan and Shen-ling-bai-zhu-San have inhibitory activities on oxidative stress [37], lipid peroxidation, and inflammatory reactions [38]. Beyond that, naringin, hesperidin, ferulic acid, and other active ingredients have some anti-inflammatory effects [36, 37]. Our results show that soothing liver and invigorating spleen recipes can protect the liver from inflammatory injury caused by an HFD, that the release of IL1 , IL-6, and TNF- $\alpha$ was significantly reduced, and that the downregulation of IL-1, IL- 6 , and TNF- $\alpha$ might be due to different degrees of inhibitory expression of IKK $\beta, \mathrm{p}-\mathrm{IKK} \beta$, and NF- $\kappa$ B. A previous study showed that the soothing liver and invigorating spleen recipes could regulate the expression and activation of the interacting protein of IKK $\beta-N F-\kappa B$ signaling pathway of KCs in NASH rats, reducing the release of inflammatory mediators (IL-6, TNF- $\alpha$, and IL-1) in KCs and, ultimately, ameliorating inflammatory damage. Consequently, a combination of the soothing liver and invigorating spleen recipes could have a significant anti-inflammatory effect, which might be closely related to their effects on the NF- $\kappa$ Bp 65 signaling pathway. 


\section{Conclusion}

In conclusion, this study showed that the release of inflammatory factors such as IL-1, TNF- $\alpha$, and IL- 6 by KCs was significantly increased by a HFD and that the IKK $\beta$-NF$\kappa \mathrm{Bp} 65$ signaling pathway maybe the effective target for the soothing liver and invigorating spleen recipes.

\section{Conflict of Interests}

All of the authors of this paper declare that they have no direct financial relation with the commercial identities mentioned in this paper. And all of the authors declare that they have no competing interests.

\section{Authors' Contribution}

Xiang-Wen Gong and Yong-Jian Xu contributed equally to this work.

\section{Acknowledgment}

The present work was supported by a Grant (no. 30973694) from the Natural Science Foundation of China.

\section{References}

[1] B. A. Elkhader and M. Z. Mahmoud, "Prevalence of nonalcoholic fatty liver among adults in Khartoum-Sudan: epidemiological survey," Journal of American Science, vol. 9, no. 6, pp. 62-66, 2013.

[2] H. Lu, H. Liu, F. Hu, L. Zou, S. Luo, and L. Sun, "Independent association between nonalcoholic fatty liver disease and cardiovascular disease: a systematic review and meta-analysis," International Journal of Endocrinology, vol. 2013, Article ID 124958, 7 pages, 2013.

[3] S. Berardis and E. Sokal, "Pediatric non-alcoholic fatty liver disease: an increasing public health issue," European Journal of Pediatrics, vol. 173, no. 2, pp. 131-139, 2014.

[4] Z. Li, J. Xue, P. Chen, L. Chen, S. Yan, and L. Liu, "Prevalence of nonalcoholic fatty liver disease in mainland of China: a metaanalysis of published studies," Journal of Gastroenterology and Hepatology, vol. 29, no. 1, pp. 42-51, 2014.

[5] W. Tomeno, M. Yoneda, K. Imajo et al., "Emerging drugs for non-alcoholic steatohepatitis," Expert Opinion on Emerging Drugs, vol. 18, no. 3, pp. 279-290, 2013.

[6] J. Xiao, K. F. So, E. C. Liong, and G. L. Tipoe, "Recent advances in the herbal treatment of non-alcoholic fatty liver disease," Journal of Traditional and Complementary Medicine, vol. 3, no. 2, pp. 88-94, 2013.

[7] K.-Q. Shi, Y.-C. Fan, W.-Y. Liu, L.-F. Li, Y.-P. Chen, and M.-H. Zheng, "Traditional Chinese medicines benefit to nonalcoholic fatty liver disease: a systematic review and meta-analysis," Molecular Biology Reports, vol. 39, no. 10, pp. 9715-9722, 2012.

[8] J. R. Liu and X. N. Zhu, "Pathogenesis and therapy summary of traditional Chinese with medicine nonalcoholic fatty liver disease," Journal of Luzhou Medical College, vol. 37, no. 6, pp. 640-641, 2014.
[9] J. X. Li, Y. L. Wang, M. Liu et al., "Treatment of nonalcoholic steatohepatitis by Jianpi Shugan Recipe: a multi-center, randomized, controlled clinical trial," Chinese Journal of Integrated Traditional and Western Medicine, vol. 34, no. 1, pp. 15-19, 2014.

[10] L. Zeng and G. G. Sheng, "The effect of Shuganjianpi and Huatanhuoxue formula on mitochondrial membrane liquidity and membrane potential level of cell model with non-alcoholic fatty liver disease," Chinese Journal of Integrated Traditional and Western Medicine on Liver Diseases, vol. 22, no. 6, pp. 360-362, 2012.

[11] J. Xiao, C. T. Ho, E. C. Liong et al., "Epigallocatechin gallate attenuates fibrosis, oxidative stress, and inflammation in nonalcoholic fatty liver disease rat model through TGF/SMAD, PI3 K/Akt/FoxO1, and NF-kappa B pathways," European Journal of Nutrition, vol. 53, no. 1, pp. 187-199, 2014.

[12] B. Wei, Q.-H. Yang, W.-J. Wang, and C.-F. Hu, "Effects of Shugan Jian-pi prescriptions on expression of IKK $\beta$ at mRNA and protein levels in liver tissues of rats with non-alcoholic steatohepatitis," Chinese Journal of Pathophysiology, vol. 28, no. 8, pp. 1448-1454, 2012.

[13] Q.-H. Yang, S.-P. Hu, Y.-P. Zhang et al., "Effect of berberine on expressions of uncoupling protein-2 mRNA and protein in hepatic tissue of non-alcoholic fatty liver disease in rats," Chinese Journal of Integrative Medicine, vol. 17, no. 3, pp. 205211, 2011.

[14] X. Y. Shi, Experimental Zoology of Modern Medicine, People's Military Medical Press, Beijing, China, 1st edition, 2000.

[15] G. F. Feng, Q. H. Yang, W. J. Wang et al., "Simultaneously isolation and identification of hepatocytes and Kupffer cells from nonalcoholic steatohepatitis rat," Guangdong Medical Journal, vol. 33, no. 1, pp. 40-43, 2012.

[16] K. M. Surapaneni and M. Jainu, "Pioglitazone, quercetin and hydroxy citric acid effect on hepatic biomarkers in Non Alcoholic Steatohepatitis," Pharmacognosy Research, vol. 6, no. 2, pp. 153-162, 2014.

[17] R. Kawamoto, K. Kohara, T. Kusunoki, Y. Tabara, M. Abe, and T. Miki, "Alanine aminotransferase/aspartate aminotransferase ratio is the best surrogate marker for insulin resistance in non-obese Japanese adults," Cardiovascular Diabetology, vol. 11, article 117, 2012.

[18] S. Fuyan, L. Jing, C. Wenjun et al., "Fatty liver disease index: a simple screening tool to facilitate diagnosis of nonalcoholic fatty liver disease in the Chinese population," Digestive Diseases and Sciences, vol. 58, no. 11, pp. 3326-3334, 2013.

[19] E. Liaskou, D. V. Wilson, and Y. H. Oo, "Innate immune cells in liver inflammation," Mediators of Inflammation, vol. 2012, Article ID 949157, 21 pages, 2012.

[20] G. Kolios, V. Valatas, and E. Kouroumalis, "Role of Kupffer cells in the pathogenesis of liver disease," World Journal of Gastroenterology, vol. 12, no. 46, pp. 7413-7420, 2006.

[21] P. J. Murray and T. A. Wynn, "Protective and pathogenic functions of macrophage subsets," Nature Reviews Immunology, vol. 11, no. 11, pp. 723-737, 2011.

[22] H. W. Zimmermann, C. Trautwein, and F. Tacke, "Functional role of monocytes and macrophages for the inflammatory response in acute liver injury," Frontiers in Physiology, vol. 3, article 56, 2012.

[23] X. Kong, N. Horiguchi, M. Mori, and B. Gao, "Cytokines and STATs in liver fibrosis," Frontiers in Physiology, vol. 3, article 69, 2012.

[24] Z. Papackova, E. Palenickova, H. Dankova et al., "Kupffer cells ameliorate hepatic insulin resistance induced by high-fat 
diet rich in monounsaturated fatty acids: the evidence for the involvement of alternatively activated macrophages," Nutrition \& Metabolism, vol. 9, article 22, 2012.

[25] G. C. Farrell, D. van Rooyen, L. Gan, and S. Chitturi, "NASH is an inflammatory disorder: pathogenic, prognostic and therapeutic implications," Gut and Liver, vol. 6, no. 2, pp. 149-171, 2012.

[26] H. Tilg, "The role of cytokines in non-alcoholic fatty liver disease," Digestive Diseases, vol. 28, no. 1, pp. 179-185, 2010.

[27] J. Zhao, H. Zheng, Y. Liu et al., "Anti-inflammatory effects of total alkaloids from Rubus alceifolius Poir. on non-alcoholic fatty liver disease through regulation of the NF- $\kappa \mathrm{B}$ pathway," International Journal of Molecular Medicine, vol. 31, no. 4, pp. 931-937, 2013.

[28] Y. Shirai, H. Yoshiji, R. Noguchi et al., "Cross talk between toll-like receptor-4 signaling and angiotensin-II in liver fibrosis development in the rat model of non-alcoholic steatohepatitis," Journal of Gastroenterology and Hepatology, vol. 28, no. 4, pp. 723-730, 2013.

[29] G. Ramakrishna, A. Rastogi, N. Trehanpati, B. Sen, R. Khosla, and S. K. Sarin, "From cirrhosis to hepatocellular carcinoma: new molecular insights on inflammation and cellular senescence," Liver Cancer, vol. 2, no. 3-4, pp. 367-383, 2013.

[30] H. L. Wright, B. Chikura, R. C. Bucknall, R. J. Moots, and S. W. Edwards, "Changes in expression of membrane TNF, NF- $\kappa$ B activation and neutrophil apoptosis during active and resolved inflammation," Annals of the Rheumatic Diseases, vol. 70, no. 3, pp. 537-543, 2011.

[31] X.-A. Wang, R. Zhang, Z.-G. She et al., "Interferon regulatory factor 3 constrains IKK $\beta / N F-\kappa B$ signaling to alleviate hepatic steatosis and insulin resistance," Hepatology, vol. 59, no. 3, pp. 870-885, 2014.

[32] J.-K. Luo, H.-J. Zhou, J. Wu, T. Tang, and Q.-H. Liang, "Electroacupuncture at Zusanli (ST36) accelerates intracerebral hemorrhage-induced angiogenesis in rats," Chinese Journal of Integrative Medicine, vol. 19, no. 5, pp. 367-373, 2013.

[33] H. F. Wei, G. Ji, and L. J. Xin, "Preliminary research on nonalcoholic fatty liver syndrome classification," Liaoning Journal of Traditional Chinese Medicine, vol. 29, no. 11, pp. 655-656, 2002.

[34] S. S. Zhang, Q. G. Li, and J. X. Li, "Diagnosis and treatment of traditional Chinese Medicine Consensus on nonalcoholic fatty liver disease," Beijing Zhong Yi Yao, vol. 29, no. 2, pp. 83-86, 2011.

[35] W. Shu-ling, J. Wei, and G. Xiao-ling, "HPLC characteristics of aqueous extract of Chaihu Shugan Powder and determination of marks," Chinese Traditional Patent Medicine, vol. 34, no. 12, pp. 2384-2388, 2012.

[36] W.-L. Li, Q.-C. Dai, Y.-B. Ji, Z. Sun, J. Du, and J. Bai, "Investigation of chemical composition and sources of Bazhentang by HPLC-ESI/MS," Chinese Pharmaceutical Journal, vol. 44, no. 21, pp. 1622-1626, 2009.

[37] Y. You, Y. H. Liu, and S. L. Gao, "Effect and mechanism of Shenling Baizhu San on the murine model of inflammatory bowel disease induced by dextran sodium sulfate in mice," Journal of Experimental Traditional Medical Formulae, vol. 18, no. 5, pp. 136-140, 2012.

[38] Z.-H. Li, J. Wang, Y.-W. Wang, R.-L. Cai, J. Sun, and M.-G. Ye, "Effect of Shenlingbaizhu powder on serum levels of EGF, SOD and MDA in ulcerative colitis rats with syndrome of dampness stagnancy due to spleen deficiency," World Chinese Journal of Digestology, vol. 20, no. 5, pp. 410-413, 2012. 


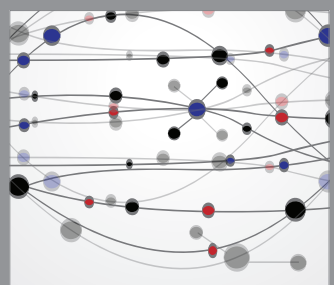

The Scientific World Journal
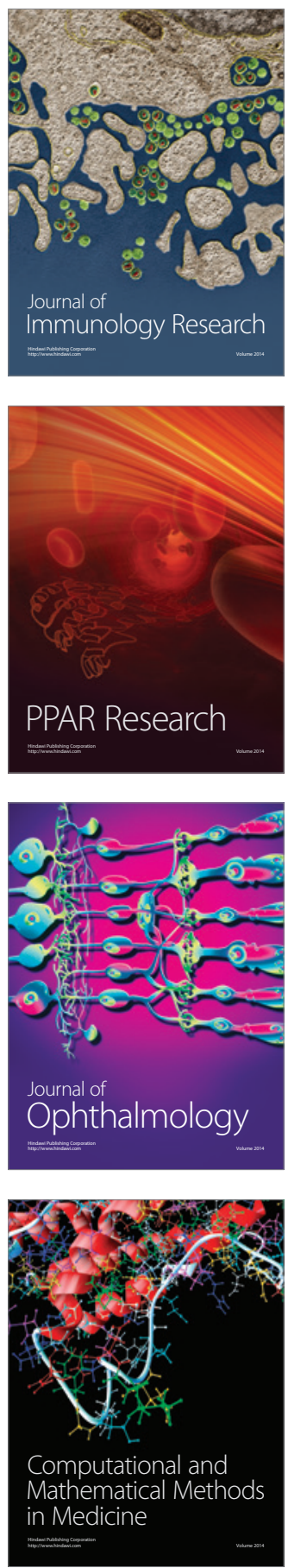

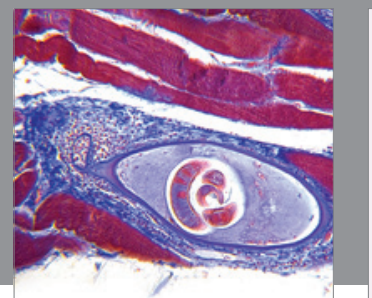

Gastroenterology

Research and Practice
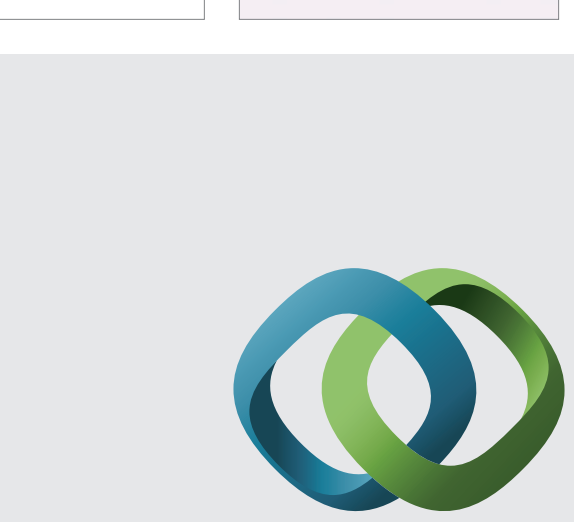

\section{Hindawi}

Submit your manuscripts at

http://www.hindawi.com
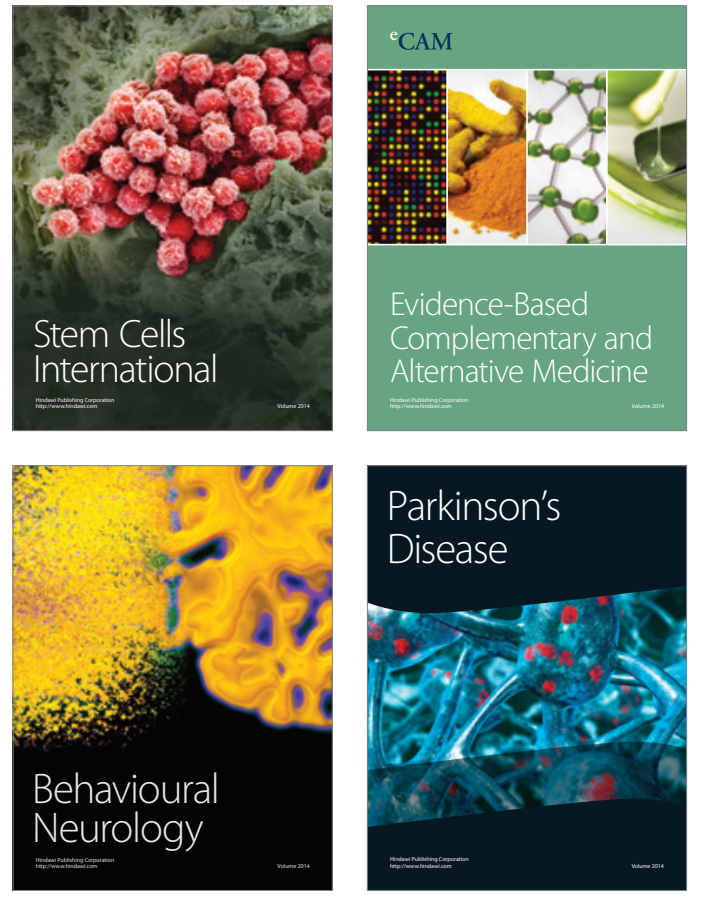
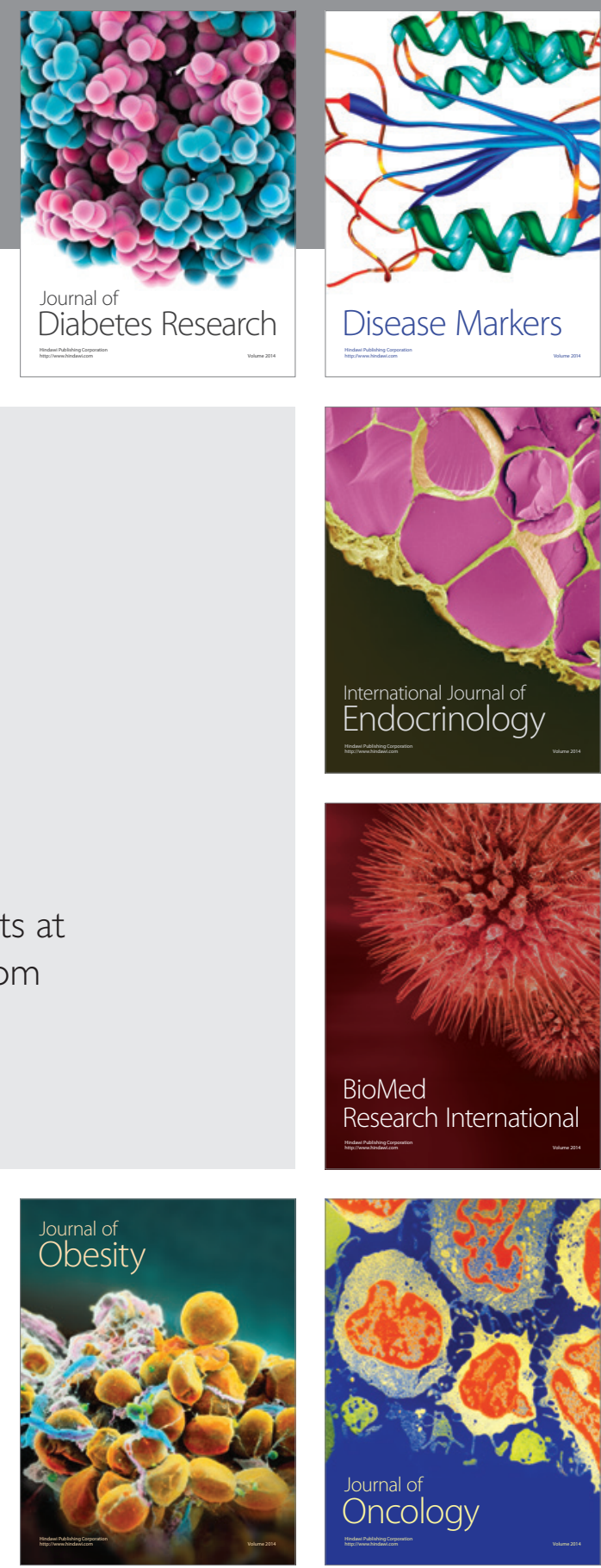

Disease Markers
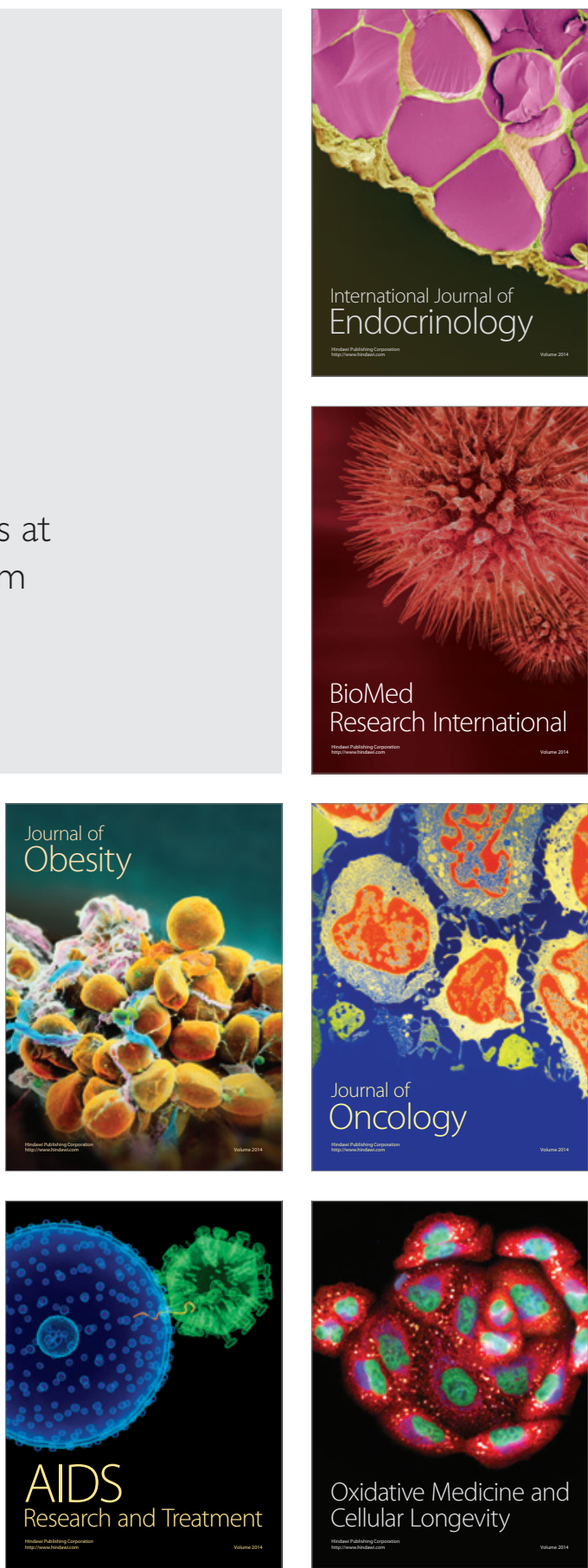